\title{
Extending the Applicability of a Newton-Kurchatov-Type Method for Solving Non-Differentiable Equations in Banach Spaces
}

\author{
Ioannis K. Argyros ${ }^{1 *}$, Santhosh George ${ }^{2}$
}

\begin{abstract}
We provide a new local convergence analysis of a Newton-Kurchatov-like method to solve non-differentiable equations in Banach spaces. Our result improve the earlier works in literature. The examples were used to test our hypotheses.

Keywords: Banach spaces, Local convergence, Newton-Kurchatov-type method, Non-differentiable equations 2010 AMS: Primary 65G99, 65H10, 65J15, 49M15.

${ }^{1}$ Department of Mathematical Sciences, Cameron University, Lawton, OK 73505, USA, iargyros@cameron.edu, ORCID:0000-0002-9189-9298 2 Department of Mathematical and Computational Sciences, National Institute of Technology Karnataka, India-575 025, sgeorge@nitk.ac.in, ORCID: 0000-0002-3530-5539

*Corresponding author: sgeorge@nitk.ac.in

Received: 4 January 2019, Accepted: 28 March 2019, Available online: 27 June 2019
\end{abstract}

\section{Introduction}

In this work, we solve

$$
H(x)=0
$$

to find a solution $x^{*} \in \Omega$, where $H: \Omega \subseteq X \longrightarrow Y$ and $X, Y$ stand for Banach spaces. Iterative methods are mostly used to solve (1.1), since solutions in closed form are hard to find. If $H$ is a differentiable operator, Newton's method is the most used method to solve the equation of $(1.1)$, which is given by [1,2]

$$
x_{n+1}=x_{n}-H^{\prime}\left(x_{n}\right)^{-1} H\left(x_{n}\right), \text { for all } n=0,1,2, \ldots x_{0} \in \Omega
$$

If $H$ is not differentiable, Remember that an operator $[x, y ; H] \in L(X, Y)$ is called a divided difference of order one for the operator $H$ on the points $x$ and $y(x \neq y)$ if the following equality holds:

$$
[x, y ; H]=H(x)-H(y) .
$$

Replacing the Fréchet derivative $H^{\prime}$ by divided differences of the operator $H$ in Newton's method (1.2) at different points, we can define two iterative methods as follows: one is the secant method is given by $[3,4]$

$$
x_{n+1}=x_{n}-\left[x_{n-1}, x_{n} ; H\right]^{-1} H\left(x_{n}\right) \quad n \geq 0, x_{0}, x_{-1} \in \Omega \text {, }
$$


and the other is Kurchatov's method given by [5]

$$
x_{n+1}=x_{n}-\left[x_{n-1}, 2 x_{n}-x_{n-1} ; H\right]^{-1} H\left(x_{n}\right) \quad n \geq 0, x_{0}, x_{-1} \in \Omega \text {. }
$$

Note that, Kurchatov's method is as simple as Newton's method and has the same rate of convergence as Newton's method. This means it has the higher rate of convergence than the Secant method. A lot of study about the convergence of Kurchatov's method have been given, see [5-10].

We split it as

$$
H(x)=F(x)+G(x)
$$

where $F: \Omega \longrightarrow Y$ and $G: \Omega \longrightarrow Y . F$ is differentiable and $G$ is continuous but non-differentiable. Then, we use the following Newton-Kurchatov-type method given by

$$
x_{n+1}=x_{n}-\left(F^{\prime}\left(x_{n}\right)+\left[x_{n-1}, 2 x_{n}-x_{n-1} ; G\right]\right)^{-1} H\left(x_{n}\right) \quad n \geq 0, x_{0}, x_{-1} \in \Omega
$$

to solve (1.1). Recently, M. A. Hernández and M. J. Rubio [8] gave an analysis of method (1.3). Cases where method (1.3) is efficient for solving systems and also arguments about its efficiency were also given in [8]. A novel idea of [8] is that the usual condition of $H^{\prime}\left(x^{\star}\right)$ is reduced to a new type condition, which means that $H$ can be a non-differentiable operator. We give a more precise local analysis for (1.3) than [8]. Advantages of our local convergence analysis over the work, in [8] :

(a) Larger radius of convergence lending to wider choice of initial guesses,

(b) More precise estimates on the distances $\left\|x_{n+1}-x^{\star}\right\|$. Hence fewer iterates are need to obtain a desired error tolerance.

(c) At least as precise information on the uniqueness ball of the solution.

These advantages are obtained under the same computational cost, since in practice the new majorizing functions are special cases of the majorizing functions in [8].

The paper is organized as follows: Section 2 contains the local convergence analysis of method (1.3). The numerical examples including favorable comparisons with earlier study [8] are presented in the concluding Section 3.

Throughout the paper we denote $B(x, \rho)=\{y \in X:\|y-x\|<\rho\}$ and $\bar{B}(x, \rho)=\{y \in X:\|y-x\| \leq \rho\}$.

\section{Local convergence analysis}

From now on by differentiable operator, we mean differentiable in the sense of Fréchet. We shall use condition $(C)$ to show the local convergence analysis of the Kurchatov-type method (1.3):

$\left(C_{1}\right) F: \Omega \subset X \rightarrow Y$ is continuously differentiable operator.

$\left(C_{2}\right) G: \Omega \subset X \rightarrow Y$ is continuous and a divided difference of order one $[\cdot, \cdot ; G]: \Omega \times \Omega \rightarrow L(X, Y)$, exists.

$\left(C_{3}\right)$ There exist $x^{\star} \in \Omega$ and $\bar{x} \in \Omega$ with $\left\|\bar{x}-x^{\star}\right\|=\delta>0$ such that $H\left(x^{\star}\right)=0$ and $M=F^{\prime}\left(x^{\star}\right)+\left[x^{\star}, \bar{x} ; G\right]$ is invertible.

$\left(C_{4}\right)\left\|M^{-1}\left(F^{\prime}(x)-F^{\prime}\left(x^{\star}\right)\right)\right\| \leq v_{0}\left(\left\|x-x^{\star}\right\|\right)$ for some function $v_{0}:[0,+\infty) \rightarrow[0,+\infty)$ continuous, nondecreasing with $v_{0}(0)=0$ and each $x \in \Omega$.

$\left(C_{5}\right)\left\|M^{-1}\left([y, 2 x-y ; G]-\left[x^{\star}, \bar{x} ; G\right]\right)\right\| \leq w_{0}\left(\left\|y-x^{\star}\right\|,\|2 x-y-\bar{x}\|\right)$ for some function $w_{0}:[0,+\infty) \times[0,+\infty) \rightarrow[0,+\infty)$ continuous, nondecreasing for each $x, y, 2 x-y \in \Omega$.

$\left(C_{6}\right)$ Equation $v_{0}(t)+w_{0}(t, \delta+t)=1$ has a minimal positive solution $\overline{r_{0}}$. Pick $r_{0} \in\left(0, \overline{r_{0}}\right]$. Define

$$
p_{0}:=v_{0}\left(r_{0}\right)+w_{0}\left(r_{0}, \delta+r_{0}\right)<1 \text {. }
$$

Let $\Omega_{0}=\Omega \cap B\left(x^{\star}, r_{0}\right)$. 
$\left(C_{7}\right)\left\|M^{-1}\left(F^{\prime}(y)-F^{\prime}(x)\right)\right\| \leq v(\|y-x\|)$ for function $v:\left[0, r_{0}\right) \rightarrow[0,+\infty)$ continuous, nondecreasing with $v(0)=0$ and all $x, y \in \Omega_{0}$.

$\left(C_{8}\right)\left\|M^{-1}\left([y, 2 x-y ; G]-\left[x^{\star}, x ; G\right]\right)\right\| \leq w\left(\left\|y-x^{\star}\right\|,\|x-y\|\right)$ for some function $w:\left[0, r_{0}\right) \times\left[0, r_{0}\right) \rightarrow[0,+\infty)$ continuous, nondecreasing for each $x, y, 2 x-y \in \Omega_{0}$.

Let $p=\int_{0}^{1} v\left(\theta r_{0}\right) d \theta+w\left(r_{0}, 2 r_{0}\right)$ and $q=\frac{p}{1-p_{0}}$.

$\left(C_{9}\right)$ Let $\bar{r} \geq 0$, there exists minimal $r \in\left(0, r_{0}\right)$ solving the equation

$$
\bar{r}=t\left[1-\frac{2\left(\int_{0}^{1} v(\theta t) d \theta+w(t, 2 t)\right)}{1-\left(v_{0}(t)+w_{0}(t, \delta+t)+2\left(\int_{0}^{1} v(\theta t) d \theta+w(t, 2 t)\right)\right)}\right] .
$$

Notice that $r>\bar{r}$.

$\left(C_{10}\right) B\left(x^{\star}, r\right) \subseteq \Omega$.

$\left(C_{11}\right)$

$$
\int_{0}^{1} v_{0}\left(\theta r^{\star}\right) d \theta+w_{0}\left(0, \delta+r^{\star}\right)<1
$$

for some $r^{*} \geq r$.

First, we need a perturbation result.

Lemma 2.1. Assume $\left(C_{1}\right)-\left(C_{6}\right)$. Then, operator $F^{\prime}(x)+[y, 2 x-y ; G]$ is invertible for all $x, y, 2 x-y \in B\left(x^{\star}, r_{0}\right)$ with $x \neq y$ and

$$
\left\|\left(F^{\prime}(x)+[y, 2 x-y ; G]\right)^{-1} M\right\| \leq \frac{1}{1-p_{0}} .
$$

Proof. Operator $[y, 2 x-y ; G]$ is well defined, since $y \neq 2 x-y$. Using $\left(C_{3}\right)-\left(C_{5}\right)$, we have in turn that

$$
\begin{aligned}
& \left\|M^{-1}\left(M-F^{\prime}(x)-[y, 2 x-y ; G]\right)\right\| \\
& \leq\left\|M^{-1}\left(F^{\prime}\left(x^{\star}\right)-F^{\prime}(x)\right)\right\|+\left\|M^{-1}\left(\left[x^{\star}, \bar{x} ; G\right]-[y, 2 x-y ; G]\right)\right\| \\
& \leq v_{0}\left(\left\|x^{\star}-x\right\|\right)+w_{0}\left(\left\|x^{\star}-y\right\|,\|\bar{x}-(2 x-y)\|\right) \\
& \leq v_{0}\left(r_{0}\right)+w_{0}\left(r_{0}, \delta+r_{0}\right)=p_{0}<1 .
\end{aligned}
$$

The result follows from the preceding estimate and the Banach lemma on invertible operators [1,2].

Secondly, we establish the sequence $\left\{x_{n}\right\}$ generated by the Newton-Kurchatov-type method (1.3) is well defined.

Lemma 2.2. Suppose the conditions $\left(C_{1}\right)-\left(C_{8}\right)$ hold, $x_{n-1}, x_{n-2}, 2 x_{n-1}-x_{n-2} \in B\left(x^{\star}, r_{0}\right)$ and $x_{n-1} \neq x_{n-2}$, then $x_{n}$ is well defined and

$$
\left\|x_{n}-x^{\star}\right\| \leq q\left\|x_{n-1}-x^{\star}\right\| .
$$

Proof. We shall use the notation

$$
M_{n-1}=F^{\prime}\left(x_{n-1}\right)+\left[x_{n-2}, 2 x_{n-1}-x_{n-2} ; G\right] .
$$

Newton-Kurchatov-type method (1.3) gives

$$
\begin{aligned}
x_{n}-x^{\star}= & x_{n-1}-x^{\star}-M_{n-1}^{-1} H\left(x_{n-1}\right) \\
= & M_{n-1}^{-1}\left(M_{n-1}\left(x_{n-1}-x^{\star}\right)-H\left(x_{n-1}\right)\right) \\
= & M_{n-1}^{-1}\left(\left(F^{\prime}\left(x_{n-1}\right)\left(x_{n-1}-x^{\star}\right)-F\left(x_{n-1}\right)+F\left(x^{\star}\right)\right)\right. \\
& \left.+G\left(x^{\star}\right)-G\left(x_{n-1}\right)+\left[x_{n-2}, 2 x_{n-1}-x_{n-2} ; G\right]\left(x_{n-1}-x^{\star}\right)\right) \\
= & -\left[M_{n-1}^{-1} M\right]\left[M^{-1} \int_{0}^{1}\left(F^{\prime}\left(x_{n-1}+\theta\left(x^{\star}-x_{n-1}\right)\right)-F^{\prime}\left(x_{n-1}\right)\right)\left(x_{n-1}-x^{\star}\right) d \theta\right] \\
& +\left[M_{n-1}^{-1} M\right]\left[M^{-1}\left(\left[x_{n-2}, 2 x_{n-1}-x_{n-2} ; G\right]-\left[x^{\star}, x_{n-1} ; G\right]\right)\left(x_{n-1}-x^{\star}\right)\right] .
\end{aligned}
$$


Using Lemma 2.1, $\left(C_{7}\right),\left(C_{8}\right)$ and the triangle inequality in the preceding identity,

$$
\begin{aligned}
\left\|x_{n}-x^{\star}\right\| \leq & \left\|M_{n-1}^{-1} M\right\|\left(\int_{0}^{1} v\left(\theta\left\|x^{\star}-x_{n-1}\right\|\right) d \theta\right. \\
& \left.+w\left(\left\|x_{n-2}-x^{\star}\right\|,\left\|x_{n-1}-x_{n-2}\right\|\right)\right)\left\|x_{n-1}-x^{\star}\right\| \\
\leq & \frac{1}{1-p_{0}}\left(\int_{0}^{1} v\left(\theta r_{0}\right) d \theta+w\left(r_{0}, 2 r_{0}\right)\right)\left\|x_{n-1}-x^{\star}\right\| \\
= & \frac{p}{1-p_{0}}\left\|x_{n-1}-x^{\star}\right\|=q\left\|x_{n-1}-x^{\star}\right\| .
\end{aligned}
$$

Let $\bar{r}=\left\|x_{0}-x^{\star}\right\|$. As in [7], [11], we must somehow drop $2 x_{n-1}-x_{n-2} \in B\left(x^{\star}, r_{0}\right)$, if $x_{n-1}, x_{n-2} \in B\left(x^{\star}, r_{0}\right)$. Suppose that $x_{1}, x_{2}, \cdots, x_{n-1} \in B\left(x^{\star}, r_{0}\right)$, then

$$
\begin{aligned}
\left\|2 x_{n-1}-x_{n-2}-x^{\star}\right\| & \leq\left\|x_{n-1}-x_{n-2}\right\|+\left\|x_{n-1}-x^{\star}\right\| \\
& \leq 2\left\|x_{n-1}-x^{\star}\right\|+\left\|x_{n-2}-x^{\star}\right\| \\
& \leq(2 q+1)\left\|x_{n-2}-x^{\star}\right\|
\end{aligned}
$$

and

$$
\left\|x_{n-2}-x^{\star}\right\| \leq q^{n-2}\left\|x_{0}-x^{\star}\right\| .
$$

Then, if $q<1$, we have

$$
\left\|x_{n-2}-x^{\star}\right\|<\left\|x_{0}-x^{\star}\right\|=\bar{r}
$$

and

$$
\left\|2 x_{n-1}-x_{n-2}-x^{\star}\right\|<(2 q+1) \bar{r} .
$$

Clearly, if $p_{0}+p<1$, then $q<1$. To show $2 x_{n-1}-x_{n-2} \in B\left(x^{\star}, r_{0}\right)$, it suffices to have $(2 q+1) \bar{r}=r$ leading to the condition $\left(C_{9}\right)$.

Theorem 2.3. Assume $(C)$ with $p_{0}+p<1$. Then, sequence $\left\{x_{n}\right\}$ generated by the Kurchatov-type method (1.3) for $x_{0} \in$ $B\left(x^{\star}, r\right)-\left\{x^{\star}\right\}$ and $x_{-1} \in B\left(x_{0}, r-\bar{r}\right)$ with $x_{-1} \neq x_{0}$ and $\bar{r}=\left\|x_{0}-x^{\star}\right\|$ exists in $B\left(x^{\star}, r\right)$, stayes in $B\left(x^{\star}, r\right)$ for all $n=0,1,2, \cdots$ and $\lim _{n \rightarrow \infty} x_{n}=x^{\star}$.

Proof. Notice

$$
\left\|x_{-1}-x^{\star}\right\| \leq\left\|x_{-1}-x_{0}\right\|+\left\|x_{0}-x^{\star}\right\| \leq r-\bar{r}+\bar{r}=r
$$

and

$$
\left\|2 x_{0}-x_{-1}-x^{\star}\right\| \leq\left\|x_{-1}-x_{0}\right\|+\left\|x_{0}-x^{\star}\right\| \leq r-\bar{r}+\bar{r}=r,
$$

so $x_{-1}, 2 x_{0}-x_{-1} \in B\left(x^{\star}, r\right)$ and $2 x_{0}-x_{-1} \neq x_{1}$. By Lemma 2.1, $x_{1}$ exists and by Lemma 2.2

$$
\left\|x_{1}-x^{\star}\right\| \leq q\left\|x_{0}-x^{\star}\right\|<\left\|x_{0}-x^{\star}\right\|=\bar{r}<r,
$$

so, $x_{1} \in B\left(x^{\star}, r\right)$ and $x_{1} \neq x_{0}$. Analogously,

$$
\begin{aligned}
\left\|2 x_{1}-x_{0}-x^{\star}\right\| & \leq\left\|x_{1}-x_{0}\right\|+\left\|x_{1}-x^{\star}\right\| \\
& \leq 2\left\|x_{1}-x^{\star}\right\|+\left\|x_{0}-x^{\star}\right\|<(2 q+1)\left\|x_{0}-x^{\star}\right\|<r,
\end{aligned}
$$

so $2 x_{1}-x_{0} \in B\left(x^{\star}, r\right)$. Assume for $k \geq 2$, if $x_{k-1}, x_{k-2} \in B\left(x^{\star}, r\right)$ for $x_{k-1} \neq x_{k-2}$, then $2 x_{k-1}-x_{k-2} \in B\left(x^{\star}, r\right)$ and hence $M_{k-1}^{-1}$ is well defined. Then, $x_{k}$ is well defined and from Lemma 2.2, we get that

$$
\left\|x_{k}-x^{\star}\right\| \leq q\left\|x_{k-1}-x^{\star}\right\| .
$$


Suppose using mathematical induction that the preceding two inequalities hold for $k=2,3, \cdots, m$, we shall show that they hold for $k=m+1$. If $x_{k}, x_{k-1} \in B\left(x^{\star}, r\right)$ with $x_{k} \neq x_{k-1}$, we get that

$$
\begin{aligned}
\left\|2 x_{k}-x_{k-1}-x^{\star}\right\| & \leq\left\|x_{k}-x_{k-1}\right\|+\left\|x_{k}-x^{\star}\right\| \\
& \leq 2\left\|x_{k}-x^{\star}\right\|+\left\|x_{k-1}-x^{\star}\right\|<(2 q+1)\left\|x_{k-1}-x^{\star}\right\| \\
& \leq(2 q+1) q^{k-1}\left\|x_{0}-x^{\star}\right\|<(2 q+1)\left\|x_{0}-x^{\star}\right\|<r,
\end{aligned}
$$

so $2 x_{k}-x_{k-1} \in B\left(x^{\star}, r\right)$ and $2 x_{k}-x_{k-1} \neq x_{k-1}$. That is by Lemma $2.1 M_{k}^{-1}$ exists and $x_{k+1}$ is well defined. Moreover, by Lemma 2.2,

$$
\left\|x_{k-1}-x^{\star}\right\| \leq q\left\|x_{k}-x^{\star}\right\|
$$

which completes the induction. That is $\left\{x_{k}\right\} \subseteq B\left(x^{\star}, r\right)$ and

$$
\left\|x_{k}-x^{\star}\right\| \leq q^{k}\left\|x_{0}-x^{\star}\right\|,
$$

from which we deduce that $\lim _{k \rightarrow \infty} x_{k}=x^{\star}$.

Next, a uniqueness result is given.

Proposition 2.4. Assume conditions $(C)$. Then, $x^{\star}$ is the only solution of equation $H(x)=0$ in $\Omega_{1}=\Omega \cap \bar{B}\left(x^{\star}, r^{\star}\right)$.

Proof. Let $y^{\star} \in \Omega_{1}$ with $H\left(y^{\star}\right)=0$. Define operator $T=\int_{0}^{1} F^{\prime}\left(x^{\star}+\theta\left(y^{\star}-x^{\star}\right)\right) d \theta+\left[x^{\star}, y^{\star} ; G\right]$. Then, using $\left(C_{4}\right),\left(C_{5}\right)$ and $\left(C_{11}\right)$.

$$
\begin{aligned}
\left\|M^{-1}(T-M)\right\| & \leq\left\|\int_{0}^{1} M^{-1}\left(F^{\prime}\left(x^{\star}+\theta\left(y^{\star}-x^{\star}\right)\right)\right) d \theta\right\| \\
& +\left\|M^{-1}\left(\left[x^{\star}, y^{\star} ; G\right]-\left[x^{\star}, \bar{x} ; G\right]\right)\right\| \\
& \leq \int_{0}^{1} v_{0}\left(\theta\left\|x^{\star}-y^{\star}\right\|\right) d \theta+w_{0}\left(\left\|x^{\star}-x^{\star}\right\|,\left\|y^{\star}-\bar{x}\right\|\right) \\
& \leq \int_{0}^{1} v_{0}\left(\theta r^{\star}\right) d \theta+w_{0}\left(0, r^{\star}+\delta\right)<1,
\end{aligned}
$$

so, $T^{-1}$ exists.

But from

$$
0=H\left(x^{\star}\right)-H\left(y^{\star}\right)=T\left(x^{\star}-y^{\star}\right)
$$

we conclude that $y^{\star}=x^{\star}$.

Remark 2.5. (a) We can set $\bar{x}=x_{0}$. In this case $\delta=\bar{r}$. let

(b) If $\Omega=X$, condition $2 x-y \in \Omega$ is automatically satisfied. To relax this condition,

$$
p_{1}=v_{0}\left(r_{0}\right)+w_{0}\left(r_{0}, \delta+3 r_{0}\right)
$$

Then, we use the condition $p_{1}+p<1$, instead of using $\left(C_{9}\right)$ to calculate $r$, or the equation

$$
v_{0}(t)++w_{0}(t, \delta+3 t)+2\left(\int_{0}^{1} v(\theta t) d t+w(t, 2 t)\right)=1 .
$$

Note that in this case $q_{1}=\frac{p}{1-p_{1}}<1$. Hence, we arrived at:

Proposition 2.6. Assume conditions $\left(C_{1}\right)-\left(C_{8}\right)$, and

$\left(C_{9}\right)^{\prime}$ There exists a solution $R \in\left(0, r_{0}\right)$ of equation (2.1) 
$\left(C_{10}\right)^{\prime}$ For $R_{1}<R, B\left(x^{\star}, R_{1}\right) \subset \Omega$

$\left(C_{11}\right)^{\prime} 2 x-y \in \Omega$ for all $x, y \in B\left(x^{\star}, R_{1}\right)$ with $x \neq y$

$\left(C_{12}\right)$

$$
\int_{0}^{1} v_{0}\left(\theta R^{\star}\right) d \theta+w_{0}\left(0, \delta+R^{\star}\right)<1
$$

for some $R^{\star} \geq R_{1}$. Then, sequence $\left\{x_{n}\right\}$ generated for $x_{0} \in B\left(x^{\star}\right)-x^{\star}$ and $x_{1} \in B\left(x_{0}, R_{1}-\bar{r}\right)$ with $x_{-1} \neq x_{0}$ by the NewtonKurchatov-type method (1.3) exists in $B\left(x^{\star}, R_{1}\right)$, stays in $B\left(x^{\star}, R_{1}\right)$ for all $n=0,1, \cdots$ and $\lim _{n \rightarrow \infty} x_{n}=x^{\star}$, which is the only solution of equation $H(x)=0$ in $\Omega_{2}=\Omega \cap \bar{B}\left(x^{\star}, R_{1}\right)$.

Remark 2.7. Clearly condition $\left(C_{11}\right)^{\prime}$ can be exchanged by

$$
\left(C_{10}\right)^{\prime \prime} B\left(x^{\star}, 3 R\right) \subseteq \Omega,
$$

since if $x, y \in B\left(x^{\star}, R\right) \Rightarrow\left\|x^{\star}-(2 x-y)\right\| \leq 2\left\|x^{\star}-x\right\|+\left\|x^{\star}-y\right\|<3 R \Rightarrow 2 x-y \in B\left(x^{\star}, 3 R\right)$ (see also [5-9] and the numerical examples).

Remark 2.8. The results in this study improve the corresponding ones in [8]. Indeed, we have the following advantages:

(1) Affine invariant results are given here which are more advantageous than non affine results given in [8].

(2) The following conditions have been used in [8]

$\left(h_{7}\right)\left\|F^{\prime}(y)-F^{\prime}(x)\right\| \leq \bar{v}(\|y-x\|)$ for all $x, y \in \Omega$,

$\left(h_{8}\right)\|[x, y ; G]-[u, v ; G]\| \leq \bar{w}(\|x-u\|,\|y-v\|)$ for all $x, y, u, v \in \Omega$,

$\left(h_{6}\right)^{\prime} \bar{p}=\gamma\left(\int_{0}^{1} \bar{v}(\theta \rho) d \theta+\bar{w}(\rho, 2 \rho)\right), \bar{q}=\frac{\bar{p}}{1-\overline{p_{0}}},\left\|M^{-1}\right\| \leq \gamma$,

$\overline{p_{0}}=\gamma(\bar{v}(\rho)+w(\rho, \rho+\delta))<1$,

$\bar{r}=t\left[1-\frac{2 \gamma\left(\int_{0}^{1} \bar{v}(\theta t) d \theta+\bar{w}(t, 2 t)\right)}{1-\gamma\left(\bar{v}(t)+\bar{w}(t \gamma, \delta+t)+2 \gamma\left(\int_{0}^{1} \bar{v}(\theta t) d \theta+\bar{w}(t, 2 t)\right)\right)}\right]$

and

$\left(h_{9}\right) B\left(x^{\star}, \rho\right) \subseteq \Omega$,

$\left(h_{10}\right)$ There exists $\rho^{\star} \geq \rho$ such that

$$
\gamma\left(\int_{0}^{1} \bar{v}\left(\theta \rho^{\star}\right) d \theta+\bar{w}\left(0, \delta+\rho^{\star}\right)\right)<1 .
$$

However, we have that

$$
\begin{aligned}
& v_{0}(t) \leq \gamma \bar{v}(t), v(t) \leq \gamma \bar{v}(t) \\
& w_{0}(s, t) \leq w(s, t) \leq \gamma \bar{w}(s, t) \\
& q \leq \bar{q} \\
& \rho \leq r
\end{aligned}
$$

and

$$
\rho^{\star} \leq r^{\star}
$$

which lead to the improvements listed in the introduction. It is worth noticing that improvements are given using the same computational cost, because in practice the computation of functions $\bar{v}, \bar{w}$ needs the computation of the functions $v_{0}, v, w_{0}, w$ as special cases. 
Remark 2.9. Let us see the radii for Newton's method (1.2), i.e., when the $\bar{v}$ and the the $\bar{v}_{0}$ functions are choosen by $\bar{v}(t)=\mu t$ and $\bar{v}_{0}(t)=\lambda t, G=0, w=w_{0}=0$ and $x^{\star}=\bar{x}$ (i.e, $\left.\delta=0\right)$. The radius $\rho$ given in [8] is

$$
\rho=\frac{2}{3 \gamma \mu} \text {. }
$$

The radius $\rho$ coincides with radius given independently by Rheinboldt [12] and Traub [13]. This value improves the radius

$$
\rho_{0}=\frac{1}{2 \gamma \mu}
$$

given also by Dennis and Schnabel $[12,13]$. Our radius of convergence $r$ is given by

$$
r=\frac{2}{\left(2 \lambda+\mu_{0}\right) \gamma}
$$

Then, we have that

$$
\rho_{0} \leq \rho \leq r
$$

The right hand side inequality in (2.2) can be strict (see (c4), (h7) and the numerical examples).

\section{Numerical examples}

Choose the divided difference $[x, y ; F]=\int_{0}^{1} F^{\prime}(y+\theta(x-y)) d \theta$.

Example 3.1. Case 1 Newton's method. Let $F, G$ be defined on $\Omega=[-1,1] \times[-1,1] \times[-1,1]$ by

$$
F(x, y, z)=\left(e^{x}-1, \frac{(e-1) y^{2}}{2}+y, z\right)^{T}, \quad \text { and } \quad G=0 .
$$

Choose $\lambda=e-1, \mu_{0}=e^{\frac{1}{e-1}}, \mu=e$ for $x^{\star}=(0,0,0)^{T}$ and $\gamma=1$

we have

$$
\rho_{0}=0.1839<\rho=0.2453<r=0.3827 .
$$

Newton's method is very efficient. In general, if the method is inefficient, then we use a better method. The new error bounds are also better, since

$$
\left\|x_{n+1}-x^{\star}\right\| \leq \frac{\mu_{0}\left\|x_{n}-x^{\star}\right\|^{2}}{2\left(1-\lambda\left\|x_{n}-x^{\star}\right\|\right)} \quad n=1,2, \ldots
$$

and

$$
\left\|x_{1}-x^{\star}\right\| \leq \frac{\lambda\left\|x_{0}-x^{\star}\right\|^{2}}{2\left(1-\lambda\left\|x_{n}-x^{\star}\right\|\right)}
$$

but $\lambda$ the old ones are given by

$$
\left\|x_{n+1}-x^{\star}\right\| \leq \frac{\mu\left\|x_{n}-x^{\star}\right\|^{2}}{2\left(1-\mu\left\|x_{n}-x^{\star}\right\|\right)}, n=0,1,2,3, \cdots .
$$

The old uniqueness ball is $B\left(x^{\star}, \frac{2}{e}\right)$. The new uniqueness ball is $B\left(x^{\star}, \frac{2}{e-1}\right)$ is better, since

$$
B\left(x^{\star}, \frac{2}{e}\right) \subseteq B\left(x^{\star}, \frac{2}{e-1}\right) .
$$

Case 2 Newton-Kurchatov-type method. Let $F$ be given as in (3.1) and define $G(x)=|x|$. We have for $\bar{x}=(0.01,0.01,0.01)^{T}, \gamma=$ $\frac{1}{2}, \delta=0.01, v_{0}(t)=(e-1) t, v(t)=e^{\frac{1}{e-1}} t, w_{0}(s, t)=w(s, t)=1, \bar{r}=\delta$ and $v_{0}(t)<v(t)$. Then

$r_{\text {old }}=0.4905<r=0.7654$. 


\section{References}

[1] I. K. Argyros, Computational theory of iterative methods, Series: Studies in Computational Mathematics 15, C.K. Chui and L. Wuytack (editors), Elservier Publ. Co. New York, USA, 2007.

[2] J.M. Ortega, W.C. Rheinbolt, Iterative Solution of Nonlinear Equations in Several Variables, Academic Press, New York, 1970.

[3] I. K. Argyros, On the Secant method, Publ. Math. Debrecen, 43 (1993), 223-238.

[4] F. A. Potra, V. Pták, Nondiscrete Induction and Iterative Methods, Pitman Publishing Limited, London, 1984.

[5] V. A. Kurchatov, On the method of linear interpolation for the solution of functional equations, (Russion) Dolk. Akad. Nauk SSSR, 1998 (1971) 524-526, translation in Soviet Math. Dolk., 12 (1971) 835-838.

[6] I. K. Argyros, On the two point Newton-like methods of convergent R-order two, Int. J. Comput. Math., 82 (2005), $219-233$.

[7] I. K. Argyros, A Kantorovich-type analysis for a fast iterative method for solving nonlinear equations, J. Math. Anal. Appl. 332 (2007), 97-108.

[8] M. A. Hernández, M. J. Rubio, On the local convergence of a Newton-Kurchatov-type method for non-differentiable operators, Appl. Math. Comput., 304 (2017), 1-9.

[9] S. Shakhno, On the Secant method under generalized Lipschitz conditions for the divided operator, PAMM-Proc. Appl. Math. Mech., 7 (2007), 2060083-2060084.

[10] A. Cordero, F. Soleymani, J. R. Torregrosa, F. K. Haghani, A family of Kurchatov-type methods and its stability, Appl. Math. Comput., 294 (2017), 264-279.

[11] I. K. Argyros, On a quadratically convergent iterative method using divided differences of order one, J. Korean. Math. S. M. E. Ser. B, 14 (3) (2007), 203-221.

[12] W. C. Rheinboldt, An adaptive continuation process for solving systems of nonlinear equations, Banach Center Publ., 3 (1977), 129-142.

[13] J. F. Traub, Iterative Methods for the Solution of Equations, Prentice Hall Englewood Cliffs, New Jersey, USA, 1994. 